\title{
Bacteriological profile with their antibiograms in neonatal septicaemia in a Teaching Hospital in Srilanka
}

\author{
Fernando R, Samarawickrama B.
}

Microbiology Department District General Hospital Chilaw

\section{Introduction}

Neonatal septicemia continues to be a major problem in Neonatal ICU. The gold standard for diagnosis of septicemia is the isolation of the microorganism from the blood culture which takes 48 hrs to 7 days. Therefore empirical treatment is crucial \& it should be based on the local sensitivity data.

\section{Objectives}

To determine the Bacteriological profile with their antibiogram and to provide sensitivity data to make local treatment protocols

\section{Materials and Methods}

Total blood culture samples received in Brain Heart Infusion broth from clinically suspected cases of neonates from neonatal ICU from 1st January, to $31^{\text {st }}$ December 2010 included for the study. Standard isolation procedures were done using blood, chocolate and MacConkey agar. All the organisms were identified by standard methods. However most Enterobactereciae were further identified using API 20E. Stokes disc diffusion method was used for susceptibility testing and ESBL detection was done using clavulanic acid .

\section{Materials and Methods}

Total blood culture samples received in Brain Heart Infusion broth from clinically suspected cases of neonates from neonatal ICU from 1st January, to $31^{\text {st }}$ December 2010 included for the study. Standard isolation procedures were done using blood, chocolate and MacConkey agar. All the organisms were identified by standard methods.

However most Enterobactereciae were further identified using API 20E. Stokes disc diffusion method was used for susceptibility testing and ESBL detection was done using clavulanic acid . 


\section{Results:}

During the study period 838 blood cultures were requested and 121 non repetitive specimens were positive (14.4\%). Gram negative bacilli (59.5\%) were more frequently isolated than Gram positive cocci (GPC) (40.5\%). API 20E identification method was able to identify Klebsiella spp (30.4\%), Enterobacter spp (20.2\%), Escherichia coli (13\%) as the common pathogens. $15.9 \%$ were unclassified with API and $20.2 \%$ of Enterobactereciae were unidentified.

Coagulase negative staphylococcus (CNS) (63\%), and Staphylococcus aureus(18\%) were the major GPC. Other pathogens were Grp D streptococcus (4), Group B streptococci

(3), Methicillin resistant Staphylococcus aureus (2), pseudomonas spp (2) and Acinetobacter spp(1).

Majority of Enterobactereciae were ESBL producers and sensitive to ciprofloxacin meropenam and amikacin . Coagulase negative stayhlococcus resistance to penicillin and cloxacillin was $100 \%$ and $83 \%$ respectively. Penicillin resistance in staphylococcus aureus was $78 \%$. However, only $18 \%$ of Staphylococcus aureus were found to be MRSA. All Gram positive organisms were sensitive to vancomycin.

Enterobactereciae antibiotic susceptibility

\begin{tabular}{|c|c|c|c|c|c|c|c|}
\hline Amp & Cefu & Co amox & Cefotax & Genta & Cipro & Merop & Amikac \\
\hline 8.3 & 13.3 & 20.6 & 38.4 & 74.1 & 95.3 & 93.3 & 100 \\
\hline
\end{tabular}

\section{Conclusion}

ESBL producing Enterobactereciae were the main pathogens in neonatal sepsis in our NICU. Empirical antibiotic choice of neonatal septicaemia in most NICUs is penicillin and cefotaxime. But High ESBL prevalence among Enterobactereciae makes cefotaxime resistant. Therefore Amikacin can be recommended as a replacement for cefotaxime for empirical treatment of sepsis to prevent mortality. This situation is serious therefore we should focus on preventive measures such as strict asepsis during labour and correct hand hygiene to prevent transmission of resistant pathogens. 